\section{GENRE}

en séries

\section{Genre en séries}

Cinéma, télévision, médias

$7 \mid 2018$

Les écrits de la réception : pratiques textuelles des publics médiatiques

\title{
Les écrits de la réception : pratiques textuelles des publics médiatiques
}

Sébastien François et Thomas Pillard

\section{(2) OpenEdition}

Journals

Édition électronique

URL : http://journals.openedition.org/ges/593

DOI : $10.4000 /$ ges.593

ISSN : 2431-6563

Éditeur

Presses universitaires de Bordeaux

Référence électronique

Sébastien François et Thomas Pillard, «Les écrits de la réception : pratiques textuelles des publics médiatiques », Genre en séries [En ligne], 7 | 2018, mis en ligne le 01 juin 2018, consulté le 18 février 2021. URL : http://journals.openedition.org/ges/593 ; DOI : https://doi.org/10.4000/ges.593

Ce document a été généré automatiquement le 18 février 2021.

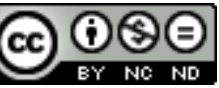

La revue Genre en séries est mise à disposition selon les termes de la Licence Creative Commons Attribution - Pas d'Utilisation Commerciale - Pas de Modification 4.0 International. 


\title{
Les écrits de la réception : pratiques textuelles des publics médiatiques
}

\author{
Sébastien François et Thomas Pillard
}

1 Comme le rappelait Marlène Coulomb-Gully en 2009, "le genre a longtemps été un point aveugle de l'analyse du discours » dans le champ académique français. Revenant cinq ans plus tard sur ce constat (Coulomb-Gully, 2014), l'auteure soulignait plusieurs avancées qui confirment la centralité et l'opérativité du genre dans les processus de communication, tout en appelant à utiliser "son extraordinaire potentiel explicatif » pour "développer les recherches sur les usages et les pratiques de réception » des publics. Une telle préoccupation a évidemment été centrale dans la création de Genre en séries : cinéma, télévision, médias et en particulier dans plusieurs articles accueillis en son sein (Ugur Tanriöver, 2015; Bertini, 2016; Olivesi, 2017), à la suite desquels s'inscrit donc le présent dossier. Cette septième livraison de la revue entend ainsi étudier les enjeux de genre qui traversent et constituent les réceptions médiatiques, en choisissant à dessein de s'intéresser aux pratiques textuelles qui accompagnent, poursuivent ou parfois anticipent ces dernières et que nous appellerons ici les « écrits de la réception ».

\section{Des réceptions actives à leurs mises en écrits}

2 Rappelons tout d'abord, pour mieux situer les visées de cette démarche dans le contexte académique français, que les études dédiées à la réception et aux publics des loisirs et de la culture y connaissent, depuis le tournant des années 2000, un essor et une diversification remarquables. Cela n'est pas sans lien avec l'écho rencontré, certes tardivement, par les cultural studies au sein de la recherche française (cf. Glevarec, 2008 ; Cervulle et Quemener, 2018 [2015]), à commencer par les travaux qui avaient renouvelé les études de réception au cours des années 1970 et 1980, en reconnaissant la part active des récepteurs/trices dans toute consommation culturelle (Hall, 1994 [1973]; Morley, 1980 ; Radway, 1984 ; Ang, 1985).

Plus largement, ce début de $\mathrm{XXI}^{\mathrm{e}}$ siècle a été propice à un dialogue transnational et pluridisciplinaire qui a permis d'articuler des traditions et des objets de recherche en 
apparence relativement éloignés. Aux études historiques et sociologiques sur les cultures littéraires populaires (Maigret, 1995; Thiesse, 2000), l'exploration des sociabilités qui entourent les consommations télévisuelles ou cinématographiques (Montebello, 1997 ; Pasquier et Jouët 1999 ; Pasquier, 1999 ; Leveratto, 2000), ou encore la construction sociale des cultes médiatiques et des communautés de fans (Le Guern, 2002) ont succédé une pluralité d'enquêtes sur les usages et les appropriations des médias de masse dans toute leur variété et leur étendue historique et géographique (Goetschel et al., 2010).

Du contenu publicitaire, musical ou vidéoludique aux célébrités des mondes artistiques, sportifs ou politiques, les contours des objets médiatiques et des pratiques qu'ils suscitent ont été redessinés dans un contexte de recompositions de leurs territoires (Ballarini et Delavaud, 2015) et du fonctionnement des industries culturelles et créatives, lié à l'essor des nouvelles technologies de l'information et de la communication ainsi qu'à la banalisation d'une culture numérique dite participative et de la convergence (Jenkins, 2006). La faculté des chercheur-e-s à revisiter d'anciennes dynamiques de production et de réception fondatrices de l'interactivité médiatique moderne (Letourneux, 2017; Absalyamova et Stiénon, 2018), tout en embrassant les innovations actuelles (Allard et Blondeau, 2007), a dès lors conduit à élargir les perspectives théoriques et à intensifier les synergies disciplinaires (Thévenin, 2015).

5 Au sein de ce paysage où les travaux sur les publics contribuent à structurer la recherche sur la « réception en actes» (François, 2013) en réaffirmant ses dimensions actives, interactives et toujours socialement situées (Juan et Trebuil, 2012 ; Poels, 2015 ; Gimello-Mesplomb et Vilatte, 2015), les pratiques textuelles au sens large (lettres à la célébrité, courriers des lecteurs/trices, analyses critiques, récits dérivés et fanfictions, etc.) sont apparues comme ayant une place incontournable, que les mutations médiatiques contribuent à revitaliser bien davantage qu'elles n'en signent l'obsolescence. Des lecteurs/trices de La Nouvelle Héloïse qui, au XVIII ${ }^{\mathrm{e}}$ siècle, ont entrepris une correspondance épistolaire avec Jean-Jacques Rousseau pour lui faire part de leurs réactions et émotions (Darnton, 1985 ; Trousson, 2011) aux fans de Lady Gaga qui répondent aujourd'hui à chacune de ses publications sur Facebook ou Twitter (Bennett, 2014), force est de constater que l'écrit, tout en s'imposant comme le médium susceptible d'œuvrer au mieux à la conservation et à la transmission des idées (KomurThilloy et Réach-Ngô, 2012), n'a (jamais) cessé d'être un vecteur privilégié pour prolonger et formaliser des expériences de réception. À l'heure de la multiplication et de la diversification des formes amateurs d'expression et de création (Jenkins, 2006 ; Allard, 2008; Flichy, 2010), le recours au texte demeure ainsi primordial pour la prise de parole, en ligne et hors ligne, de toute une partie des récepteurs/trices. Face aux difficultés guettant le/la chercheur/se en quête des "sens des publics " (Céfaï et Pasquier, 2003), à l'ère contemporaine comme pour des périodes antérieures, il s'avère dès lors essentiel de rappeler et de souligner le « réel avantage » qu'il y a de disposer de traces de réception directement produites par les personnes ou groupes de personnes concernés (François, 2013), y compris et peut-être surtout lorsqu'il s'agit d'analyser ces traces hétérogènes au prisme du genre. 


\section{Des pratiques d'écriture genrées ?}

6 Les pratiques d'écriture fournissent effectivement des ressources précieuses pour traiter des problèmes théoriques, méthodologiques et épistémologiques liés à l'étude du «genre de la réception» (Biscarrat, 2015), comme l'attestent les jalons posés par l'ensemble des travaux fondateurs dans ce domaine évoqués ici. Par-delà les contenus qui leur donnent naissance, les différences de contexte socio-historique ou les dispositifs de communication mobilisés, les matériaux textuels analysés et dans une certaine mesure « inventés " par les chercheur-e-s - qu'ils soient issus d'archives, de recherches ethnographiques et/ou de collectes numériques -, en disent souvent long sur leurs auteur-e-s comme sur la composition et le degré de structuration des publics auxquels ils appartiennent (Abercrombie et Longhurst, 1998 ; Céfaï et Pasquier, 2003). Ils sont, à ce titre, un des principaux lieux où se jouent et se révèlent les enjeux de genre qui façonnent aussi bien ces différents publics que les interactions sociales qu'ils négocient à partir des contenus qui suscitent l'écriture.

7 Les écritures « ordinaires », « domestiques » ou « non-scolaires » étant déjà hautement genrées (Blanc, 1993 ; Lahire, 1997 ; Lyons, 2013), il apparait fécond d'analyser le profil des rédacteurs/trices des multiples types de textes adossés à la réception d'objets culturels eux-mêmes porteurs de représentations et de normes genrées. L'étude des conditions d'écriture et, le cas échéant, de publication s'avère tout autant fondamentale pour préciser les usages de tels écrits à des échelles individuelles ou collectives. Dans le prolongement, par exemple, de travaux sur les appropriations différenciées de la lecture (Long, 2003; Albenga, 2011 et 2017) ou sur la construction des identités - adolescentes, masculines, féminines, LGBT, etc. - à l'aide des médias de masse (Kearney, 2006 ; Cann, 2014 ; Hilton-Morrow et Battles, 2015), de telles enquêtes permettent de comprendre les modes de présentation de soi, les implications idéologiques ou encore les perceptions des rapports de genre qui s'élaborent à travers ces pratiques rédactionnelles, sans les dissocier de l'environnement social où elles s'insèrent.

8 Le regain de visibilité et d'intérêt actuel des questions de genre dans la culture et les médias, consécutif aux mouvements \#metoo et \#balancetonporc de la fin d'année 2017, confirme aussi bien à ce titre la pertinence du projet proposé par ce numéro - initié en juillet 2016 - que son actualité et sa spécificité vis-à-vis de la littérature existante. La pénétration du genre dans les études françaises de réception s'est en effet traduite récemment par des recherches ambitieuses et originales, explorant des terrains jusquelà peu ou pas fréquentés, tels que les croisements entre gender et fan studies (Bourdaa et Alessandrin, 2017), la place du genre dans la critique d'art (Buscatto et al., 2017) ou l'essor des pratiques féministes en ligne (Blandin 2017; Blandin et al., 2017; Pahud et Paveau, 2017).

9 Si on ne peut qu'espérer que ces entreprises stimulantes se prolongeraient in fine par d'autres travaux, elles demeurent encore peu fréquentes. Envisager de façon transversale et globale la réception (textuelle) des objets médiatiques en mobilisant un approche genrée éclaire pourtant sous un jour nouveau tant les contenus consommés que les discours et les pratiques qu'ils suscitent, que ces derniers émanent d'ailleurs des récepteurs/trices les plus impliqué-e-s, à l'image des "fans", ou des plus « ordinaires ». De même, analyser de près les écrits de réception et ouvrir la voie à leur comparaison ne peut que faire émerger de nouvelles pistes de travail, susceptibles de 
nous renseigner sur les logiques qui travaillent le genre autant que sur les manières dont celles-ci peuvent évoluer historiquement et culturellement. Tel est le pari de ce numéro, qui s'attache à mettre en regard les analyses de matériaux hétérogènes, produits à des époques et dans des contextes variés, tout en faisant dialoguer des recherches francophones et anglophones.

\section{Présentation du numéro}

L'article de Delphine Chedaleux apporte tout d'abord un aperçu inédit sur les usages sociaux du cinéma en France dans les années 1940 et 1950, à partir d'écrits autobiographiques de jeunes spectateurs ou spectatrices. S'appuyant sur ces traces de la réception ordinaire de plusieurs films de la période, l'auteure dévoile de manière nuancée comment ces derniers ont pu participer à la construction de soi des individus, entre expérimentation des normes genrées dans la pratique spectatorielle et réflexivité sur sa propre identité de genre et son rapport au désir.

11 La deuxième contribution est issue du travail de Jules Sandeau sur la réception du film Drive (Nicolas Winding Refn, 2011) sur le site spécialisé Allociné, où le long-métrage a suscité des commentaires pour le moins contradictoires: le discours de cinéphiles autoproclamés y est révélateur de multiples représentations genrées, qui se trouvent mobilisées pour discréditer les spectateurs/trices qui n'auraient su apprécier le film à sa juste valeur. L'étude s'attache à les répertorier ainsi qu'à montrer leur articulation avec des représentations de classe, de génération ou encore de sexualité.

Linda Chance offre ensuite un panorama des productions textuelles qui commentent un genre de programme spécifique à la télévision japonaise publique depuis les années 1960, les asadora, ces feuilletons matinaux destinés à une audience majoritairement féminine. Croisant les réactions professionnelles et amateures sur différents supports, la chercheuse analyse comment parler des asadora produit des formes d'énonciation marquées plutôt par le masculin, quel-le-s qu'en soient d'ailleurs les auteur-e-s, et met en évidence le caractère paradoxal des réceptions de fans au sein de cette textualité : l'enjeu y est moins la discussion des normes traditionnelles présentes dans ces programmes que le développement de sociabilités autour de la répétition d'un plaisir partagé.

13 Signé par Jonathan A. Rose, le quatrième article pose une pierre importante dans l'étude des fanfictions en ligne dans la perspective des études de genre en discutant les "transfics", c'est-à-dire les histoires écrites par des fans qui mettent en scène des personnages ouvertement transgenres, à partir de celles dérivées de la série télévisée Sherlock (2010-). Ce type de récit, qui n'est évidemment pas sans lien avec d'autres souscatégories de fanfictions bousculant déjà les frontières de genre, ouvre sur des problématiques spécifiques telles que la représentation de soi des individus transgenres et le statut des corps transgenres, à distance des modèles, de surcroît encore rares, proposés par les médias.

Enfin, Hélène Breda souligne comment les écrits de la réception peuvent s'infiltrer dans d'autres formes de créativité faniques, à l'image des fanarts, ces dessins inspirés par toute sorte de contenus médiatiques existants: ici, des images mettant en scène les princesses Disney voient leur sens remodelé par l'ajout de phrases ou de phylactères, dans une direction qui semble pouvoir être qualifiée de féministe au sens large du terme. L'auteure étudie les caractéristiques de ces matériaux hybrides, apparus en plus 
grand nombre sur Internet depuis la fin des années 2000, où cohabitent des messages qui s'attaquent directement aux stéréotypes de genre présents dans les œuvres originales et d'autres plus généraux dans la lutte contre le patriarcat.

Si chacun de ces articles apporte des connaissances nouvelles sur les écrits de la réception, c'est aussi de leur juxtaposition qu'émergent des réflexions fécondes et de nouvelles pistes de recherche. Loin d'être exhaustifs, nous en proposons trois cidessous, qui font également le lien avec la littérature existante et les domaines liés aux pratiques textuelles des publics restés relativement absents parmi les propositions reçues et dans la version finale de ce dossier.

\section{Des textes entre consommation et participation culturelle}

Les articles de ce numéro rappellent à quel point les écrits de la réception ont été et demeurent variés. Cela ne tient toutefois pas seulement aux formats choisis (court, long; papier, en ligne, etc.) ou aux types de discours mobilisés (critique, narratif, parodique, etc.) : le recours à l'écrit engage les récepteurs et réceptrices concerné-e-s sur la voie, plus ou moins poussée, d'une objectivation de leurs propres consommations culturelles, où peuvent émerger des enjeux liés au genre de multiples manières.

La richesse de certains témoignages autobiographiques - mêmes incidents, comme au détour de journaux intimes ou de correspondances privées - a depuis longtemps été perçue par les recherches, notamment historiques, qui ont pu s'en saisir pour mieux connaître les lecteurs - puis les lectrices - des périodes passées (cf. Brouard-Arends, 2003), ou plus récemment les spectateurs et spectatrices de cinéma (voir l'article de Delphine Chedaleux dans ce numéro). À l'autre bout du spectre, des écrits plus spécifiques s'inscrivent explicitement dans le prolongement de réceptions, en développant des critiques plus ou moins argumentées d'œuvres ou de produits culturels : si l'on peut évidemment s'intéresser à ce qu'elles disent du genre et des rapports de genre dans les médias, il est aussi essentiel de voir qu'elles sont ellesmêmes souvent marquées par des fractures liées au genre, selon les lieux où elles s'expriment - des fanzines papier (Duncombe, 1997; Hein, 2006) à Internet (Pasquier, 2014) - et qui les écrit, entre critique professionnelle et critique "profane » ou " amateure ». Une telle perspective informe dans ce numéro les contributions de Jules Sandeau, qui explore ainsi les représentations genrées portées par les commentaires postés sur le site Allociné, et de Linda Chance qui étudie l'entrelacement de ces différentes formes de critique autour des asadora. N'oublions pas également les réactions peut-être plus affectives, spontanées - pour ne pas dire épidermiques - qui tentent d'entrer en interaction avec les protagonistes des contenus médiatiques, qu'ils les créent ou les incarnent. Les individus qui s'adressent à leurs idoles, à des auteur-e-s, voire à des personnages de fiction - par courrier ou via des réseaux sociaux en particulier - présentent souvent des caractéristiques sociales singulières (et notamment de genre) ou invitent à réfléchir au statut et à la fonction des relations "parasociales» au regard des représentations genrées sur les publics médiatiques. C'est en tout cas ce que montrent les lettres adressées par toutes ces petites filles aux personnages du feuilleton Hélène et les garçons dans les années 1990 (Pasquier, 1999) ou les travaux récents dédiés au courrier reçu par les vedettes de cinéma dès les années 1920 (Juan, 2014). Il serait par conséquent souhaitable que ces matériaux rares, mais 
extrêmement riches, soient davantage exploités par les chercheur-e-s, à l'image des tweets échangés entre stars et internautes dont les celebrity studies ont repéré le potentiel (Marwick et Boyd, 2011 ; Click et al., 2013).

En somme, les publics maîtrisant l'écriture ont toujours cultivé des pratiques, voire des stratégies, pour exprimer et développer leur rapport émotionnel ou analytique à certains objets médiatiques, en allant parfois jusqu'à proclamer la nature de ce rapport dans la sphère publique. Les échanges épistolaires (principalement entre lectrices) et la " lettre au grand écrivain » ont par exemple contribué à forger entre la fin du XVIII ${ }^{\mathrm{e}}$ et le milieu du XIX siècle un régime de communication littéraire et un système d'évaluation des œuvres qui demeurent au cœur des pratiques contemporaines (Diaz, 1994 ; Lyon-Caen, 2006). De l'autre côté de l'Atlantique, le public adolescent et féminin américain a joué un rôle majeur dans l'essor d'une cinéphilie de masse ainsi que dans l'institutionnalisation du star-system, à travers l'envoi de courriers aux journaux, aux studios et aux vedettes (Anselmo-Sequeira, 2015). De nos jours enfin, la grande majorité des blogs de critique de cinéma ou de séries restent tenus par des hommes, dont les logiques d'action individuelles accompagnent les mutations contemporaines du marché promotionnel des images animées (Dupuy-Salle, 2014). Entre consommation et participation, les écrits de la réception - et les dynamiques genrées qui leur sont propres - prennent donc part au fonctionnement et à l'économie de certains champs de la culture, ce qui les expose aussi à d'éventuelles récupérations de la part des industries culturelles.

\section{Écrire les politiques de l'identité}

19 Ce numéro envisage également les pratiques textuelles comme des instruments de construction de soi, pouvant œuvrer à la création d'espaces d'autonomie, d'apprentissage ou d'émancipation pour les identités féminines comme pour les identités masculines, queer, etc. Se prêtant aussi bien à des approches historiques que culturelles, une telle perspective a donné lieu à des travaux explorant la «fabrique de l'intime» (Seth, 2013) au travers de l'écriture, aussi bien que des formes plus engagées de revendications identitaires, lorsque les écrits discutent par exemple de pratiques sexuelles taboues ou même juridiquement condamnables (Ferreday, 2015).

La transmission écrite de l'acte de réception peut alors se faire outil de politisation des rapports sociaux en offrant à une partie des récepteurs/trices l'opportunité de faire entendre leur voix dans des arènes traversées par des conflits de définition et des luttes entre discours hégémoniques et contre-hégémoniques autour d'identités genrées (Maigret et Macé, 2005). Il est ainsi pertinent d'examiner la manière dont des « contrepublics subalternes » (Fraser 1990) tentent, à travers la médiatisation de leur parole et l'appropriation de certains objets culturels, de négocier ou de performer leurs identités (de genre, sexuelle, ethnique, de classe, etc.), de débattre des normes dominantes - en particulier, celles présentes dans ces contenus qui les inspirent - ou encore de redéfinir leur place dans la sphère publique. Des travaux rattachés aux fan studies ont notamment souligné comment certaines catégories de fanfictions remettent en cause ou à l'inverse renforcent l'hétéronormativité des relations amoureuses dans nos sociétés (Jenkins, 1992; Tosenberger, 2008), tandis que d'autres enquêtes révèlent combien certains textes de fans s'inscrivent dans le "fan activism ", c'est-à-dire le soutien à des causes 
telles que le combat contre les violences faites aux femmes en ayant pour support des contenus médiatiques populaires (Jenkins et Shresthova, 2012).

En écho à ces enjeux, plusieurs textes de ce numéro discutent des potentialités démocratiques et politiques de ces foyers discursifs en se demandant s'ils peuvent être perçus, vécus ou médiatisés, selon les cas : comme des «techniques de soi » (Foucault, 1982), en ce qui concerne les autobiographies et journaux intimes étudiés par Delphine Chedaleux, comme des "écritures de la différence» (Zoberman, 2008) pour ce qui est des «transfic» auxquels s'intéresse Jonathan A. Rose, ou comme des "pratiques militantes » (Blandin, 2017), au sujet des fanarts «féministes » analysés par Hélène Breda. En miroir, d'autres recherches, comme celle de Linda Chance ici, rappellent que les potentialités subversives des réceptions actives ne vont pas de soi et sont toujours à évaluer au cas par cas.

Des travaux complémentaires seraient dès lors à initier afin de déterminer à quelles conditions les écrits de la réception ont pu participer, dans une variété de contextes socio-historiques et médiatiques spécifiques, à l'édification et à la transmission de cultures des minorités ou de la marginalité, particulièrement du point de vue des questions de genre et de sexualité. Une telle démarche permettrait non seulement de renforcer la visibilité et la connaissance de ces politiques discursives et identitaires, mais aussi de mener des recherches comparatives et diachroniques : on pourrait ainsi se demander, en particulier, si la présence sur Internet de groupes sociaux dominés contribue significativement à changer la donne et à influer positivement sur les puissants processus de normalisation à l'œuvre dans les productions culturelles (Djavadzadeh et Raboud, 2016).

\section{Pratiques collectives de l'écriture et représentations des publics médiatiques}

Tandis que la prise en compte des sociabilités et des dimensions collectives des consommations culturelles s'est ancrée enfin plus solidement au sein des études de réception, notamment en France, depuis le tournant des années 2000 ( $c f$. Gire et al., 2007), un autre enjeu de ce numéro est de rappeler que toute réception est loin d'être purement personnelle et idiosyncrasique. Même seul-e face à sa feuille ou à son écran, les écrivant-e-s savent généralement que d'autres font ou ont fait l'expérience du même objet culturel, ce qui n'est pas sans conséquence sur leurs productions écrites.

Au regard de ces jeunes fans d'Hélène, de la série Hélène et les garçons, qui lui écrivaient en sachant pertinemment que leur lettre surnagerait difficilement au milieu de milliers d'autres (Pasquier, 1999) ou du courrier des lecteurs de certains magazines de cinéma des années 1950, qui révèlent l'existence et les riches interactions de publics cinéphiles féminins (Sellier, 2009), les écrits de la réception sont une belle invitation à réfléchir à la manière dont ces textes témoignent de l'existence de collectifs imaginés (et parfois fantasmés), comme le "public», les "bons» ou les "mauvais» fans, etc. Dans ce dossier, la contribution de Jules Sandeau est par exemple révélatrice des aprioris qui pèsent encore sur la figure du "fan", mise à distance par d'autres internautes dits " cinéphiles", ce qui contraste avec les évolutions récentes qui participent de leur réhabilitation, ou tout au moins de leur mainstreamisation (cf. Bennett et Booth, 2016). 

protagonistes bien plus tangibles, en publiant par exemple sur des supports dont ils/ elles ne sont que les usagers et dont ils/elles subissent les règles et les aléas : nous pensons ainsi au travail de sélection et/ou d'édition de l'«homme-réponse » du courrier des lecteurs d'un magazine (Charpentier 2003; Pillard 2015) ou à celui des modérateurs de forums communautaires. Les rédacteurs/trices peuvent en outre subir la censure de certains lieux de publication ou bien, à l'inverse, être partie prenante des organisations collectives qui concourent à façonner et à diffuser leurs textes, ce qui s'observe, entre autres, du côté des fanfictions, cas d'école pour l'analyse d'une écriture collaborative majoritairement féminine (Bacon-Smith 1992; François 2009; Jamison 2013), ou encore dans la production en ligne de certains fans des asadora étudiée par Linda Chance dans ce numéro.

ecrits liés à des réceptions sont par conséquent toujours à replacer dans le cadre d'interactions, de coopérations et éventuellement de frictions entre les participant-e-s à ces «mondes» de l'écrit (Becker, 1988), notamment quand y interviennent des dynamiques genrées. Il en découle fréquemment des conventions d'écriture et/ou des catégorisations amateurs, qui ne sont justement pas étrangères aux identités, voire aux stéréotypes de genre: ici, le rejet de certains marqueurs féminins dans les commentaires des asadora qu'observe Linda Chance, ou la stabilisation de codes autour des fanarts de princesse Disney que décrit Hélène Bréda, en sont de claires illustrations. Ce repérage des normes liées au genre dans ces matériaux hétérogènes s'avère alors utile pour comprendre comment les modes d'appréciation des produits culturels se stabilisent ou évoluent, à l'image de ce que l'on peut repérer dans les pratiques textuelles cinéphiles (Jullier et Leveratto 2010; Pasquier et al., 2014) ou sériephiles (Combes 2011), en participant (ou non) des transformations des rapports de genre.

Le chantier des écrits de la réception semble ainsi encore largement à ouvrir ou à poursuivre, même s'il s'appuie aujourd'hui sur une littérature en réalité fournie bien qu'elle soit éclatée, et donne lieu régulièrement à des travaux passionnants, ce dont ce dossier donnera, nous l'espérons, un aperçu supplémentaire. Les textes étudiés, qu'ils aient eu vocation à être publiés ou à rester dans le for privé, donnent en effet à voir une partie des processus qui contribuent directement à la construction des identités de leurs auteur-e-s où les contenus culturels de tous ordres jouent un rôle crucial: de cette manière, si l'on commence de mieux en mieux à connaître comment les réceptions médiatiques façonnent l'identité des adolescentes et préadolescentes (Kearney, 2006; Duits et van Romondt Vis, 2009), le travail reste encore largement à faire pour de nombreuses autres identités fondées sur le genre et la sexualité. L'exploration des pratiques textuelles aura sans nul doute à y contribuer.

\section{BIBLIOGRAPHIE}

ABERCROMBIE Nicholas et Brian LONGHURST (1998), Audiences. A Sociological Theory of Performance and Imagination, Londres, Sage Publications Ltd.

Genre en séries, 7 | 2018 
ABSALYAMOVA Elina et Valérie STIÉNON (2018), La Voix du lecteur dans la presse française au XIX ${ }^{e}$ siècle, Limoges, Presses Universitaires de Limoges et du Limousin.

ALBENGA Viviane (2017), S'émanciper par la lecture. Genre, classe et usages sociaux des livres, Rennes, Presses Universitaires de Rennes.

ALBENGA Viviane (2011), « Stabiliser ou subvertir le genre ? Les effets performatifs de la lecture », Sociologie de l'art, n 17, 2011, p. 31-43. URL : https://www.cairn.info/revue-sociologie-de-lart-2011-2-page-31.htm

ALLARD Laurence (2008), « L'impossible politique des communautés à l'âge de l'expressivisme digital », Sens Public, vol. 3, n 7-8. URL : https://www.cairn.info/revue-cahiers-senspublic-2008-3-page-105.htm

ALLARD Laurence et Olivier BLONDEAU (2007), MédiaMorphoses, $\mathrm{n}^{\circ} 21$ « 2.0 ? Culture numérique, cultures expressives ».

ANDREJEVIC Mark (2008), "Watching Television Without Pity : the Productivity of Online Fans », Television New Media, vol. 9, n 1, DOI : 10.1177/1527476407307241

ANG Ieng (1985), Watching Dallas. Soap Opera and the Melodramatic Imagination, Londres et New York, Routledge.

ANSELMO-SEQUEIRA Diana (2015), «Screen-Struck : The Invention of the Movie Girl Fan ». Cinema

Journal, vol. 55, n 1, p. 1-28. DOI : 10.1353/cj.2015.0067

BACON-SMITH Camille (1992), Enterprising Women. Television Fandom and the Creation of Popular Myth, Philadelphie, University of Pennsylvania Press.

BALLARINI Loïc et Gilles DELAVAUd (dir.) (2015), Nouveaux territoires médiatiques, Paris, Mare \& Martin.

BECKER Howard S. (1988), Les Mondes de l'art, Paris : Flammarion.

BENNETT Lucy (2014), « 'If we stick together we can do anything' : Lady Gaga fandom, philanthropy and activism through social media », Celebrity Studies, vol. $5 ; n^{\circ} 1-2$. URL : https:// www.tandfonline.com/doi/abs/10.1080/19392397.2013.813778

BENNETT Lucy et Paul воотн (dir.) (2016), Seeing Fans. Representations of Fandom in Media and Popular Culture, New York, Bloomsbury.

BERTINI Marie-Joseph (2016), « Genre 2.0. Le Web, un champ structuré en domination. Vers une économie politique des pratiques langagières en ligne ", Genre en séries : cinéma, télévision, médias, $\mathrm{n}^{\circ} 3$, « Dynamique des études de genre appliquées aux objets médiatiques ». URL : http:// genreenseries.weebly.com/uploads/1/1/4/4/11440046/3_bertini.pdf

BISCARRAT Laetitia (2015), « Le genre de la réception. Stéréotypes de genre et fictions sérielles », Communication, vol. 33, $\mathrm{n}^{\circ}$ 2. URL : https://journals.openedition.org/communication/5775

BLANC Dominique (1993), « Correspondances. La raison graphique de quelques lycéennes », dans David BucKingham (dir.), Reading Audiences. Young People and the Media, Manchester : Manchester University Press.

BLANDIN Claire (dir.) (2017), Réseaux, n²01, « Féminisme en ligne ». URL : https://www.cairn.info/ revue-reseaux-2017-1.htm

BlANDin Claire, Sandrine LeVeque, Simon MASSEI et Bibia PAVARd (dir.) (2017), Le Temps des médias, $\mathrm{n}^{\circ} 29$, « Féminismes ». URL : https://www.cairn.info/revue-le-temps-des-medias-2017-2.htm 
BOURDAA Mélanie et Arnaud ALESSANDRIN (dir.) (2017), Fan Studies/Gender Studies. La rencontre, Condé-sur-Noireau : Téraèdre.

BROUARD-ARENDS Isabelle (dir.) (2003), Lectrices d'Ancien Régime, Rennes, Presses Universitaires de Rennes.

BUSCATTO Marie, Mary LEONTSINI et Delphine NAUDIER (dir.) (2017), Du genre dans la critique d'art / Gender in Art Criticism, Paris, Éditions des archives contemporaines.

CANN Victoria (2014), « The Limits of Masculinity : Boys, Taste and Cultural Consumption », dans Steven ROBERTS (dir.), Debating Modern Masculinities. Change, Continuity, Crisis ?, Basingstoke et New York, Palgrave Macmillan, p. 17-34.

CEFAï Daniel et Dominique PASQUIER (dir.) (2004), Les Sens du public. Publics politiques, publics médiatiques, Paris, Presses Universitaires de France.

CERVULLE, Maxime et Nelly QUEMENER (2018 [2015]), Cultural Studies. Théories et méthodes, Paris, Armand Colin.

CHARPENTIER Émilie (2003), «Spectateurs, vous avez la parole ! Le courrier des lecteurs dans Cinémagazine et Mon Ciné », Mémoire de Maîtrise en histoire sous la direction de Pascal ORY et Christian-Marc BosséNo, Université Paris 1 - Panthéon-Sorbonne.

CLICK Melissa A., Hyunji LEE et Holly Willson HolladAy (2013), « Making Monsters : Lady Gaga, Fan Identification, and Social Media », Popular Music and Society, vol. 36, n 3, p. 360-379.

COMBES Clément (2011), « La consommation de séries à l'épreuve d'internet. Entre pratique individuelle et activité collective », Réseaux, nº 165, p. 137-63. DOI : 10.3917/res.165.0137 COULOMB-GULLY Marlène (2014), « Inoculer le Genre. Le Genre et les SHS : une méthodologie traversière ", Revue française des sciences de l'information et de la communication, $\mathrm{n}^{\circ} 4$, « Recherches au féminin dans les sciences de l'information et de la communication ». URL : https:// journals.openedition.org/rfsic/837

COULOMB-GUlly Marlène (2009), « Les sciences de l'information et de la communication : une discipline gender blind?", Questions de communication, $\mathrm{n}^{\circ}$ 15. URL : https://

journals.openedition.org/questionsdecommunication/518

DARNTON Robert (1985), Le Grand massacre des chats. Atttitudes et croyances dans l'ancienne France, Paris, Robert Laffont.

DIAZ José-Luis (dir.) (1994), Textuel, n² 27, « Écrire à l'écrivain »

DJAVADZADEH Keivan et RABOUd Pierre (dir.), Raisons politiques, $\mathrm{n}^{\circ}$ 62, « Pop et populaire. Politiques du mainstream », Presses de Science Po, 2016. URL : https://www.cairn.info/revue-raisonspolitiques-2016-2.htm

DUNCOMBE Stephen (1997), Notes from Underground. Zines and the Politics of Alternative Culture, Londres, Verso.

DUITS Linda et Pauline VAN ROMONDT VIS (2009), « Girls make sense : Girls, celebrities and identities ", European Journal of Cultural Studies, vol. 12, $\mathrm{n}^{\circ}$ 1, p. 41-58.

DUPUY-SALLE Manuel (2015), « La dimension communicationnelle des cinéphilies ordinaires contemporaines : quatre logiques d'actions individuelles à l'œuvre dans l'animation de blogs ", dans Jean-Paul AUBERT et Christelle TAILLIBERT (dir.), Les Nouvelles pratiques cinéphiles, Paris :

L'Harmattan. 
FERREDAY Debra (2015), « Game of Thrones, Rape Culture and Feminist Fandom », Australian Feminist Studies, vol. $30, \mathrm{n}^{\circ} 83$.

FLICHY Patrice (2010), Le Sacre de l'amateur. Sociologie des passions ordinaires à l'ère numérique, Paris, Éditions du Seuil/La République des idées.

FOUCAULT Michel (2001), Dits et écrits (1954-1988), tome IV : 1980-1988, Paris, Gallimard.

FRASER Nancy (1990), « Rethinking the Public Sphere : A Contribution to the Critique of Actually Existing Democraty », Social Text, $n^{\circ}$ 25/26, p. 56-80.

FRANÇOIS Sébastien (2013), «Les créations dérivées comme modalité de l'engagement des publics médiatiques : le cas des fanfictions sur Internet », Thèse de doctorat en Sciences économiques et sociales sous la direction de Dominique Pasquier, Télécom Paris Tech.

FRANÇOIS Sébastien (2009), « Fanf(r)ictions. Tensions identitaires et relationnelles chez les auteurs de récits de fans ", Réseaux, n 153, p. 157-89. URL : https://www.cairn.info/revuereseaux-2009-1-page-157.html

GIMELLO-MESPlomb Frédéric et Jean-Christophe VilatTe (dir.) (2015), Revue française des sciences de l'information et de la communication, $\mathrm{n}^{\circ} 7$, « Les recherches sur les publics en Sciences de l'Information et de la Communication ». URL : https://journals.openedition.org/rfsic/1462 GIRE Fabienne, Dominique PASQUIER et Fabien GRANJON (2007), « Culture et sociabilité. Les pratiques de loisirs des Français », Réseaux, vol. 25, nº 145-146, p. 159-215. URL : https://www.cairn.info/ revue-reseaux1-2007-6-page-159.htm

GLEVAREC Hervé, Éric MACÉ Et Éric MAIGRet (2008). Cultural Studies : Anthologie, Paris, Armand Colin. GOETSCHEL Pascale, JOST François et Myriam TSIKounAs (dir.) (2010), Lire, voir, entendre. La réception des objets médiatiques, Paris, Éditions de la Sorbonne.

HALL Stuart (1994 [1973], « Codage/décodage », Réseaux, n 68, p. 29-39. URL : https:// www.cairn.info/revue-reseaux1-1994-6-p-27.htm HEIN Fabien (2006), « Le critique rock, le fanzine et le magazine : 'Ça s'en va et ça revient'«, Volume !. La revue des musiques populaires, 2006, vol. 5, n 1, p. 83-106.

HILTON-MORROW Wendy et Kathleen BATTLES (2015), Sexual Identities and the Media. An Introduction, New York et Londres, Routledge.

HUNTER Jane H. (2003), How Young Ladies Became Girls. The Victorian Origins of American Girlhood. New Haven, Yale University Press.

JAMISON Anne (2013), Fic. Why Fanfiction Is Taking over the World, Dallas (Texas), Smart Pop.

JENKINS Henry et Sangita SHRESTHova (2012), « Up, Up, and Away! The Power and Potential of Fan Activism », Transformative Works and Cultures, n 10. DOI : https://doi.org/10.3983/twc.2012.0435 JENKINS Henry, 2006, Convergence Culture. Where Old and New Media Collide, New York, New York University Press, 2006.

JENKINS Henry (1992), Textual Poachers. Television fans \& Participatory Culture, New York, Routledge. JUAN Myriam (2014), « « Encore une admiratrice! » À quoi sert une star de cinéma : réflexion à l'aune de lettres de spectateurs et de spectatrices (début des années 1920, début des années 1930) », Mise au point. Cahiers de l'association française des enseignants et chercheurs en cinéma et audiovisuel, $\mathrm{n}^{\circ}$ 6. URL : https://journals.openedition.org/map/1684 
JUAN Myriam et Christophe trebuil (2012) (dir.), Conserveries mémorielles, $\mathrm{n}^{\circ} 12$, «Publics de cinéma : pour une histoire des pratiques sociales. URL : http://cm.revues.org/1067

JULLIER Laurent et Jean-Marc LEVERATTO (2010), Cinéphiles et cinéphilies. Une histoire de la qualité cinématographique, Paris, Armand Colin.

KEARNEY Mary Celeste (2006), Girls Make Media, New York, Routledge

KOMUR-THILLOY Greta et Anne RÉACH-NGÔ (dir.) (2012), L'Écrit à l'épreuve des médias du Moyen Âge à l'ère électronique, Paris : Classiques Garnier.

LAHIRE Bernard (1997), « Masculin-féminin. L'écriture domestique », dans Daniel Fabre (dir.), Par écrit. Ethnologie des écritures quotidiennes, Paris, Maison des Sciences de l'Homme, p. 145-61.

LE GUERN Philippe (dir.) (2002), Les Cultes médiatiques. Cultures fan et cuvres cultes, Rennes, Presses Universitaires de Rennes.

LETOURNEUX Matthieu (2017), Fictions à la chaîne. Littératures sérielles et culture médiatique, Paris, Le Seuil.

LEVERATTO Jean-Marc (2006), Introduction à l'anthropologie du spectacle, Paris, La Dispute.

LONG Elizabeth (2003), Book Club. Women and the Uses of Reading in Everyday Life, Chicago, University of Chicago Press.

LYON-CAEN Judith (2006), La Lecture et la vie. Les usages du roman au temps de Balzac, Paris, Tallandier. LYONS Martyn (2013), The Writing Culture of Ordinary People in Europe, c.1860-1920, Cambridge, Cambridge University Press.

MAIGRET Éric et Éric MACÉ (dir.) (2005), Penser les médiacultures. Nouvelles pratiques et nouvelles approches de la représentation du monde, Paris, Armand Colin et Institut national de l'audiovisuel.

MAIGRET Éric (1995). « 'Strange grandit avec moi'. Sentimentalité et masculinité chez les lecteurs de bandes dessinées de super-héros ", Réseaux, vol. 13, n 70, p. 79-103.

MARWICK Alice et Danah Boyd (2011), " To See and Be Seen : Celebrity Practice on Twitter », Convergence, vol. 17, $\mathrm{n}^{\circ}$ 2, p. 139-158.

MORLEY David (1980), The Nationwide Audience, Structure and Decoding, Londres, British Film Institute.

MONTEBELLO Fabrice (1997), « Spectacle cinématographique et classe ouvrière, Longwy (1944-1960) », thèse de doctorat en histoire sous la direction de Yves Lequin et Heinz-Gerhard Haupt, Université Lyon 2.

OLIVESI Aurélie (2017), « ‘Ces parents sont de vrais tarés !' L’opposition à une expérience de parentalité alternative dans les commentaires en ligne comme structuration d'une grammaire anti-genre ", Genre en séries : cinéma, télévision, médias, $\mathrm{n}^{\circ} 6$, « Être père, être mère : représentations et discours médiatiques ». URL : http://genreenseries.weebly.com/uploads/ 1/1/4/4/11440046/6.2_olivesi.pdf

PASQUIER Dominique, Réseaux, $n^{\circ}$ 183, «Évaluations profanes : la critique en ligne ». URL : https:// www.cairn.info/revue-reseaux-2014-1.htm

PASQUIER Dominique (1999), La Culture des sentiments. L'expérience télévisuelle des adolescents, Paris, Maison des Sciences de l'Homme. 
PASQUIER Dominique et Josiane JOUËT (dir.) (1999), Réseaux, vol. 17, n 92-93, « Les jeunes et l'écran. Enquête nationale auprès des 6-17 ans ». URL : https://www.cairn.info/revue-reseaux1-1999-1p-25.htm

PAHUD Stéphanie et Marie-Anne PAVEAU (dir.) (2017), Argumentation et analyse du discours, $\mathrm{n}^{\circ} 18$, « Nouvelles argumentations féministes ». URL : https://journals.openedition.org/aad/2301

PILLARD Thomas (2015), « Cinéphilie populaire et usages sociaux du cinéma dans les années 1950 : le courrier des lecteurs du Film complet (1949-1958) ", Studies in French Cinema, vol. 15, n 1, p. 69-87.

POELS Géraldine (2015), Les Trente glorieuses du téléspectateur. Une histoire de la réception télévisuelle des années 1950 aux années 1980, Bry-sur-Marne : INA Éditions, 2015.

RADWAY Janice (1984), Reading the Romance. Women, Patriarchy, and Popular Litterature, Chapel Hill, University of North Carolina Press.

SELLIER Geneviève (2009), «Le courrier des lecteurs de Cinémonde dans les années 1950 : la naissance d'une cinéphilie au féminin », dans Noël BURCH et Geneviève SELLIER, Le Cinéma au prisme des rapports de sexe, Paris, Vrin, p. 67-90.

SETH Catriona (2013), La Fabrique de l'intime. Écrits autobiographiques de femmes du XVIIIe siècle, Paris, Robert Laffont.

THÉVENIN Olivier (2015), « Publics, médias de masse et participation culturelle. Trois concepts interdépendants ", Revue française des sciences de l'information et de la communication, $\mathrm{n}^{\circ} 4$. URL : https://journals.openedition.org/rfsic/1541

THIESSE Anne-Marie (2000), Le Roman du quotidien. Lecteurs et lectures populaires à la Belle Époque, Paris : Le Chemin vert.

TOSENBERGER Catherine (2008), « Homosexuality at the Online Hogwarts : Harry Potter Slash Fanfiction », Children's Literature, $n^{\circ} 36$, p. 185-207.

TROUSSON Raymond (2011), Lettres à Jean-Jacques Rousseau sur la Nouvelle Héloïse, Paris, Champion.

UĞUR TANRIÖVER Hülya (2015), « Femmes turques et séries télévisées : diversité des lectures, richesse des usages », Genre en séries : cinéma, télévision, médias, $\mathrm{n}^{\circ} 1$, « Les séries euroméditerranéennes à l'épreuve du genre ». URL : http://genreenseries.weebly.com/uploads/ 1/1/4/4/11440046/ges_n\%C2\%B01_tanri\%C3\%B6ver.pdf

ZOBERMAN Pierre (dir.) (2008), Queer. Écritures de la différence ?, 2 tomes, Paris, L'Harmattan.

\section{AUTEURS}

\section{SÉBASTIEN FRANÇOIS}

Sébastien François est docteur en sociologie et agrégé de sciences économiques et sociales, rattaché au laboratoire EXPERICE (Université Paris 13). Ses travaux portent sur les pratiques culturelles des jeunes, en particulier numériques, les industries culturelles médiatiques (dessin animé, applications mobiles, etc.), de leur réception à leur production, et les cultures fans. Outre de nombreux articles sur ces sujets, il a dirigé avec Gilles Brougère L'Enfance en conception(s). Comment les industries culturelles s'adressent-elles aux enfants? (Peter Lang, 2018). 


\section{THOMAS PILLARD}

Thomas Pillard est maître de conférences en études cinématographiques et audiovisuelles à l'Université Sorbonne Nouvelle - Paris 3, membre de l'IRCAV et du comité éditorial de Genre en séries. Associant histoire culturelle et études culturelles, ses recherches portent sur l'histoire du cinéma en France, les relations entre cinémas populaires et cultures médiatiques (genres, stars, presse spécialisée) ainsi que l'étude des réceptions et des publics audiovisuels dans leur diversité. Il a notamment publié Le Film noir français face aux bouleversements de la France d'après-guerre,

1946-1960 (Joseph K, 2014), Bertrand Tavernier - Un dimanche à la campagne (Atlande, 2015), et dirigé Le Film français : rôles, fonctions et identités d'une revue corporative (Théorème $n^{\circ} 20, \mathrm{PSN}, 2015$, avec L. Creton et K. Kitsopanidou). 